\title{
Research and Application of A Variable Step-size ALE Algorithm Used in Echo Detection of Active Sonar
}

\author{
Linfeng JIANG ${ }^{1, a}$, Shengzeng ZHOU $^{1, b}$, Delong SUN ${ }^{1, c}$ \\ ${ }^{1}$ No.5200, JinDu Road, Minhang District, Shanghai, China \\ apeter_2500@163.com, bview222@sina.com, 'dlong726@sina.com
}

Keywords: ALE, Variable Step-size, LMS Algorithm, Echo Dectection.

\begin{abstract}
Narrow-band signal or CW pulse can be effectively detected by ALE algorithm. ALE based on LMS algorithm is widely used. The convergence speed and convergence accuracy of fixed step-size LMS algorithm is a pair of contradictions. The non-stationary data must be normalized before it is applied. The local reverberation background may be enhanced when the original data is normalized, so the echo detection is unfavorably affected. In order to solve the problem, a variable step-size LMS algorithm based on power estimation has been researched. Through the simulation and analysis of sea trial data, it is proven that reverberation background level can be reduced effectively using the variable step-size LMS algorithm. The output SNR of target echo can be enhanced by 8dB, the display quality can be effectively improved.
\end{abstract}

\section{Introduction}

Adaptive Line Enhancer(ALE) is a kind of adaptive filter technique, which is widely used in signal processing[1]. And it is a typical application of adaptive noise canceller. The narrow-band signal or CW pulse signal with unknown frequency buried in noise background can be effectively detected by ALE algorithm[2]. The structure of ALE is simple and its principle is intelligible. It is easy to be applied practically without extra referenced input data.

The CW pulse is a frequently-used waveform for detection of moving target in active sonar system. The detection background is weak reverberation or noise for Doppler effect of moving target. The background noise can be cancelled by ALE. So the output SNR and display quality of CW pulse can be effectively improved.

The step-size of common LMS algorithm is fixed, so the convergence speed and convergence accuracy of fixed step-size LMS algorithm is a pair of contradictions[3,4,5]. Stationary input data is required for the algorithm. But the received data of active sonar is usually non-stationary because of reverberation. So it is necessary to normalize the input data before using the LMSALE. In order to avoid the unfavorably influence of normalization, a variable step-size LMS algorithm based on power estimation has been proposed in echo detection of active sonar.

\section{The Principle of ALE Algorithm}

The basic principle of ALE algorithm is that a delay element is inserted to the input mixed signal, which is used to de-correlate the noise component, while the CW pulse signal is still correlated because of its periodic property. The adaptive filter is a narrow-band filter centered at the frequency of the sinusoidal component. So the noise component is weakened, while the sinusoidal component is enhanced.

Fixed Step-size LMSALE. The principle block diagram of a fixed step-size LMSALE is shown in Fig. 1. The kernel part is an adaptive noise canceller. The main input $x(k)$ is mixed by sinusoidal signal $s(k)$ and noise signal $n(k)$. The input delay $\Delta$ is considered as a reference input of the canceller. The length of the delaying line is denoted by symbol $\Delta$. The condition of $\Delta>1 / B$ must be fulfilled, in which $B$ is the bandwidth of the noise, $N$ is the length of the transversal filter. 


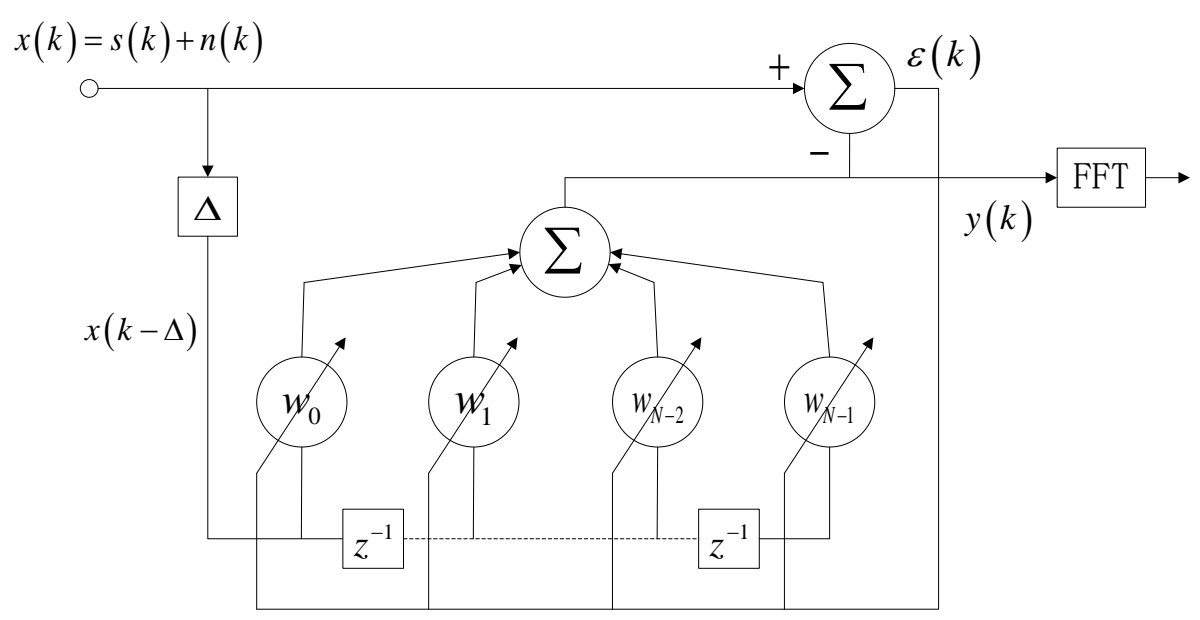

Fig.1 Principle Block Diagram of LMSALE

The iterative algorithm of the LMSALE is given by

$$
y(k)=\sum_{i=0}^{N-1} w_{i}(k) x(k-\Delta-i)
$$

Where, $N$ is the length of the adaptive filter, which is related to the pulse length of CW. The tap weights vector of the filter and input vector are denoted by $w(k)$ and $x(k)$ respectively. The delay points of noise de-correlation is denoted by symbol $\Delta$, which is related with the bandwidth of the noise.

$\varepsilon(k)$ is the output estimation error of ALE

$$
\varepsilon(k)=x(k)-y(k)
$$

The LMS iterative algorithm of the tap weights vector is given by

$$
w_{i}(k+1)=w_{i}(k)+\mu \varepsilon(k) x(k-\Delta-i)
$$

And the initial condition is

$$
w_{i}(0)=0, i=0,1, \ldots, N-1
$$

In Eq. 3, $\mu$ is the adaptive iterative step-size. The LMS algorithm is used in the iterative process. The value $\mu$ needs a balanced choice between convergence speed and the minimum error. And it should be properly chosen through simulation and practical data processing.

The capability of ALE is decided by filter length $N$ and adaptive step-size $\mu$. We can achieve as satisfying filtering capability as possible by adjusting the two parameters on condition that the power of the input background noise has been normalized.

Variable Step-size NLMSALE. The convergence capability of the adaptive algorithm is embodied in how to choose the step-size $\mu$. The common LMS algorithm has a fixed step-size $\mu$. If the step-size $\mu$ is small, the algorithm has a small misalignment rate, high convergence accuracy and low convergence speed. In opposite, the algorithm has a big misalignment rate, low convergence accuracy and high convergence speed[6]. The convergence speed and convergence accuracy of fixed step-size LMS algorithm is a pair of contradictions. In order to deal with the contradiction, a lot of methods have been proposed by researchers. The NLMS algorithm is a kind of important variable 
step-size algorithm, by which the best convergence speed and satisfying steady-state response can be obtained. The main equations are described as below.

$$
\begin{aligned}
& w(k+1)=w(k)+\mu(k) \varepsilon(k) x(k) \\
& \mu(k)=\frac{\alpha}{N \hat{P}_{i}(k)} \quad, \quad 0<\alpha<2
\end{aligned}
$$

Where, $\hat{P}_{i}(k)$ represents the power estimation of signal $x(k)$ at time instant $k . N$ is the length of the filter. An exponential window is used to estimate the power of $x(k)$. The equation is given by

$$
\hat{P}_{i}(k)=(1-\beta) P_{i}(k)+\beta x^{2}(k)
$$

Where, the smoothing factor is represented by symbol $\beta$, which meets the condition of $0<\beta<1$. A smaller value of $\beta$ is chosen if the signal is stationary. In opposite, a bigger value of $\beta$ is chosen to trail the change of the input power. The advantages of the algorithm are fast convergence speed and good steady-state capability. The step-size is calculated by power estimation of the input signal, and the step-size $\mu$ can be changed automatically according to the input power estimation.

\section{Simulation and Essentials Analysis of ALE Algorithm}

The simulation block diagram of ALE is shown in Fig. 2.

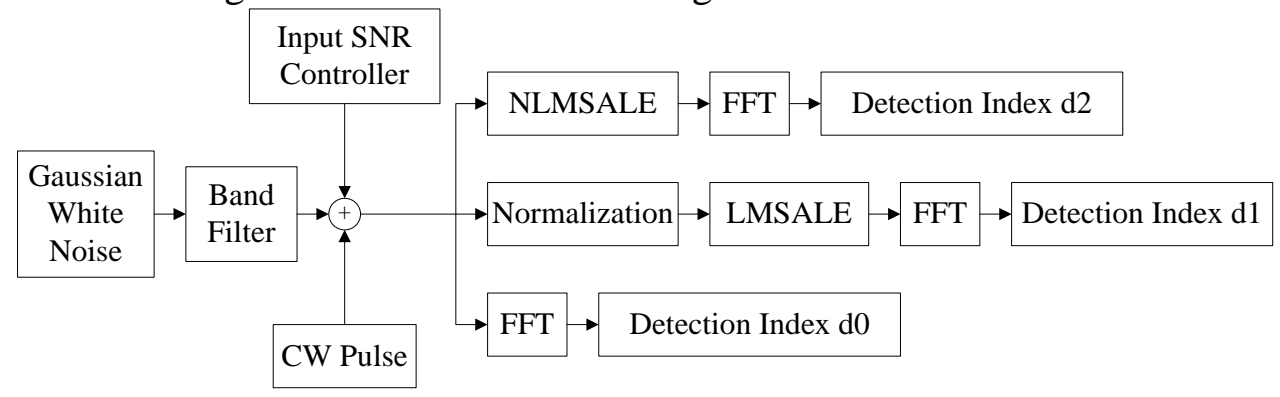

Fig.2 Simulation Block Diagram of ALE

The basic conditions of the simulation are shown as followed. The frequency of CW signal is $f_{0} \mathrm{~Hz}$. The bandwidth of noise is $f_{0} \pm 1000 \mathrm{~Hz}$. The input SNR is defined by the ratio of power of the CW signal to power of the noise with frequency $1 \mathrm{~Hz}$. The input SNR is corresponded to the detection index $d$.

Normalization. Because the step-size of common LMSALE is fixed, it is necessary to make the input signal stationary. The power of active sonar data is not stationary because the reverberation level and the echo strength of the target both change a lot due to different distances. So the input signal is necessary to be normalized. And its principle block diagram is shown as below.

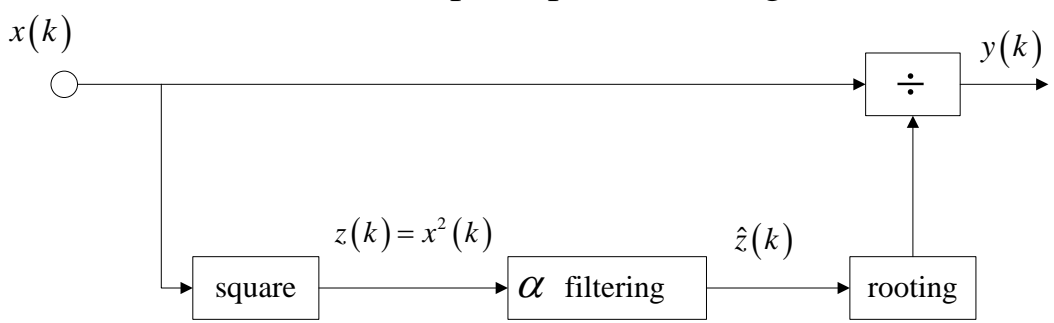

Fig.3 Principle Block Diagram of Normalization

The alpha filter principle are denoted by

$$
z(k)=x^{2}(k)
$$




$$
\hat{z}(k+1)=\hat{z}(k)+\frac{1}{M}[z(k+1)-\hat{z}(k)]
$$

And the initial condition is given by

$$
\hat{z}(0)=z(0)
$$

The time constant $M$ is described by equation $M=10 T f s, T$ is the pulse length, which needs to be optimized in practical calculation. The ratio of signal to reverberation can't be affected by normalization, the echo wave structure can't be affected either.

Essentials Analysis. The capability of ALE algorithm is mainly affected by adaptive step-size and length of adaptive filter. Here the simulation manner is used to optimize the two parameters by comparing the output SNR(detection index). Consider the input SNR 32dB, the detection index is $18 \mathrm{~dB}$ in theory. The signal is delayed by $10 / B$, the search range of the adaptive step-size is $1 e-11 \sim 1 e-4$. The length of the adaptive weight is $0.05 T \sim 0.5 T$.

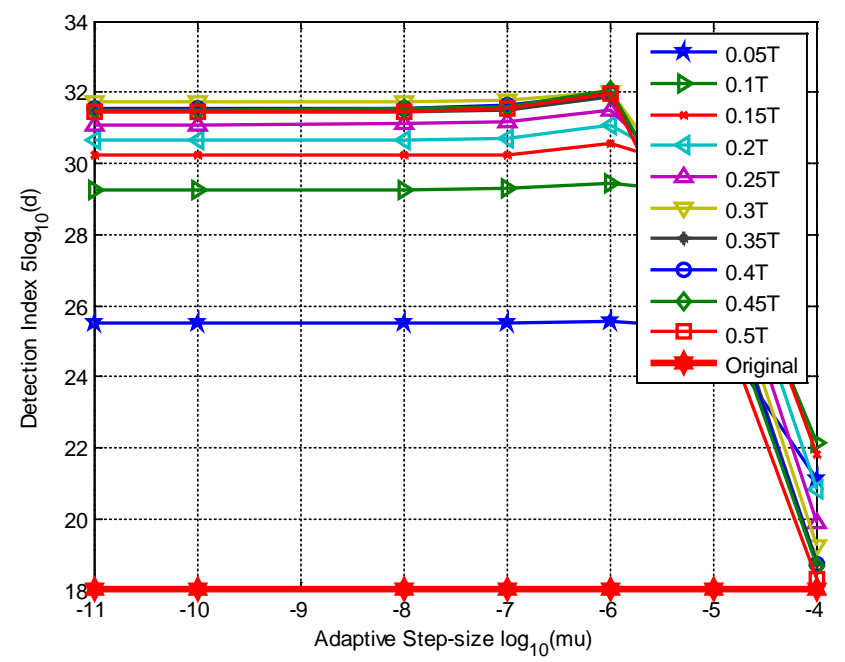

Fig.4 Output Detection Index with Different Adaptive Step-size and Length of Filter

The output detection index with different adaptive step-size and different length of filter is given in Fig. 4. From which, the following conclusions can be summarized.

(a).The smaller step-size, the higher output SNR. But the capability is changed little after the step-size is smaller than the value $1 e-7$.

(b). The extent of the capability's improvement is pretty much the same on condition that the length of the filter is chosen between $0.2 T \sim 0.5 T$. The parameters must be optimized in practical situations according to the pulse width of signal and the calculation cost.

\section{Data Processing and Analyzing of Sea Trial}

The subplot 1 of Fig. 5 is the waveform of original in time domain. The amplitude of the signal is not limited. But the input waveform is obviously non-stationary because of the reverberation and the TVG. The subplot 2 of Fig. 5 is the waveform after filtering. The target can be obviously detected in time domain because the target echo is strong. The subplot 3 of Fig. 5 is the normalized waveform, which meets the stationary demand before LMSALE. The subplot 4 of Fig. 5 is the waveform after LMSALE. The signal is not obvious because of the enhancement of the reverberation background caused by normalization. The subplot 5 of Fig. 5 is the waveform after NLMSALE. The level of background has obviously been reduced and the output SNR of signal has been enhanced obviously. 

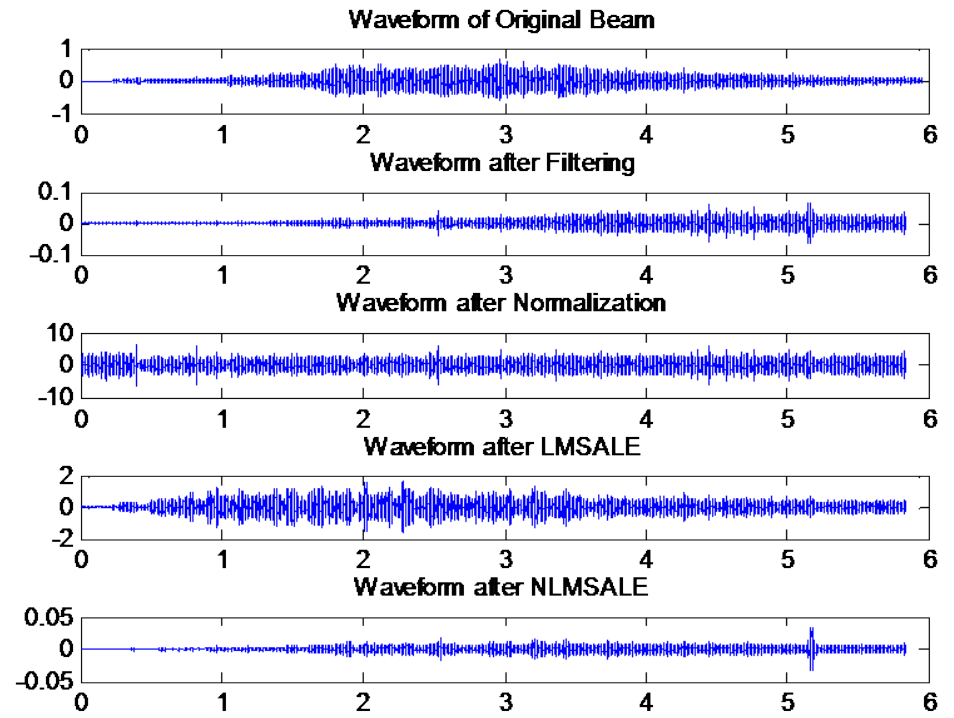

Fig.5 Output Waveforms of Each Stage

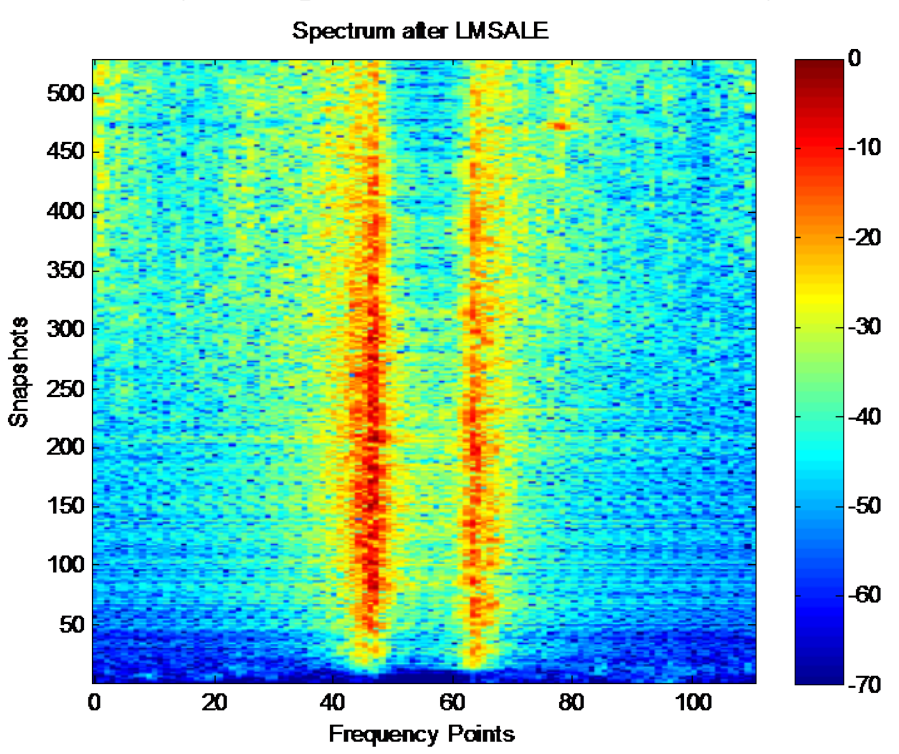

Fig.6 Spectrum after LMSALE

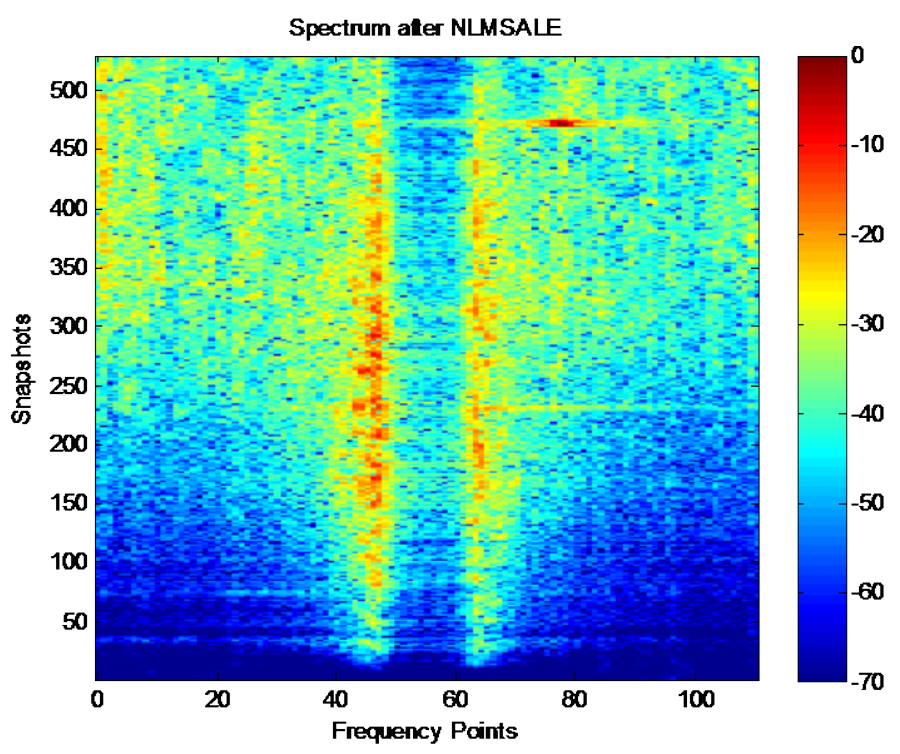

Fig.7 Spectrum after NLMSALE 
The output spectrum of LMSALE with fixed step-size is shown in Fig. 6. In the figure, it is easy to find that the noise background near the target echo has been cancelled obviously. But the reverberation can't be cancelled by LMSALE. The level of reverberation in a near distance is still strong. And it is stronger than original because of normalization. This is the main disadvantage of LMSALE algorithm.

In order to overcome the disadvantage of LMSALE, it is considered to use the NLMSALE with variable step-size, the result is shown in Fig. 7. Compared with Fig. 6, we can easily find that the noise background has been cancelled obviously, the target echo is more clear. And the output SNR is enhanced observably. The detection quality of CW pulse can be effectively improved.

\section{Conclusions}

ALE can be used to detect a narrow-band signal or CW pulse signal with unknown frequency buried in noise background effectively. The practical data received by active sonar is not stationary because of multiple factors such as reverberation strength in different distance, the strength of target echo and the TVG curve. The level of the signal is much different from distances and it is hard to distinguish the differences between different frequencies effectively by normalization in time domain. So the LMSALE algorithm with fixed step-size doesn't work well in the practical data processing. However, the NLMSALE algorithm can be used to overcome the above disadvantages. It works well in practical data processing. The output SNR of target can be enhanced by $8 \mathrm{~dB}$, and the detection quality of CW pulse can be effectively improved.

\section{References}

[1] Simon Haykin. Adaptive Filter Theory[M].Fourth Edition. Translated by Zheng Baoyu. Publishing House of Electronics Industry, Beijing.2006.

[2] HOU Baochun, HUI Junying and CAI Ping. Performance improvement of adaptive line enhancer using coherent accumulative algorithm[J]. ACTA ACUSTICA,vol.16(1),p25,Jan.1991.

[3] R.H.Kwong and E.W.johnston. A variable step size LMS algorithm[J]. IEEE Trans Signal processing,vol.40,p1663-1642,July 1992.

[4] GE Huamin, ZHAO Weiwei. Improved variable step-size and variable parameters LMS adaptive filtering algorithm[J]. Sensors \& Transducers,vol.158(11),p369-373,2013.

[5] ZHANG Wenwen, SI Xicai, CHAI Juanfang, LI Li. New variable step size LMS adaptive spectral-line enhancement algorithm[J]. System Engineering and Electronics, vol.31(1), p33,Jan. 2009.

[6] LI Pingping, PEI Bingnan and HU Lijun. Improved adaptive LMS algorithm[J]. IEEE Trans Computer Engineering and Applications,vol.40(13),p134,2011. 